\title{
THE CONORMAL MODULE OF AN ALMOST COMPLETE INTERSECTION
}

\author{
ERNST KUNZ
}

\begin{abstract}
The conormal module of an ideal $I$ in a commutative ring $S$ is the $S / I$-module $I / I^{2}$. Assume $S$ is a regular noetherian ring and $I$ a prime ideal, which is locally everywhere a complete intersection or an almost complete intersection (i.e. needs one generator more than in the complete intersection case). In this situation necessary and sufficient conditions for $I / I^{2}$ being torsion free are given. Moreover the torsion of $I / I^{2}$ is expressed in terms of Kähler differentials of $S / I$.
\end{abstract}

1. Torsion freeness of the conormal module. Let $S$ be a regular local ring, $I$ an ideal of $S$ and $R=S / I$. We say that $I$ (or $R$ ) is a "complete intersection", if $\mu(I)=\mathrm{ht}(I)$, and that $I$ (or $R$ ) is an "almost complete intersection", if $\mu(I)=\mathrm{ht}(I)+1$. Here $\mu$ denotes the minimal number of generators and ht means "height".

$I$ is a complete intersection iff the conormal module $I / I^{2}$ is a free $R$-module (see [3] or [9]). In this note we are interested in necessary and sufficient conditions for $I / I^{2}$ being torsion free, in case $I$ is a prime ideal and an almost complete intersection. Observe that for a prime ideal $I$ the $R$ module $I / I^{2}$ is torsion free iff $I^{2}$ is an $I$-primary ideal.

THEOREM 1. Let $S$ be a regular noetherian ring, I a prime ideal of $S$ which is locally everywhere a complete intersection or an almost complete intersection. For $R=S / I$ let $K_{R}$ be the canonical (dualizing) module of $R$, i.e. $K_{R}=$ $\operatorname{Ext}_{S}^{r}(R, S)$, where $r=\mathrm{ht}(I)$. Then the following conditions are equivalent:

(a) $I / I^{2}$ is a torsion free $R$-module.

(b) $K_{R}$ is a reflexive $R$-module.

(c) For all $P \in \operatorname{Spec}(R)$ with $\mathrm{ht}(P)=1$ the local ring $R_{P}$ is a complete intersection.

From this we see, for example, that if under the assumptions of the theorem we have $\operatorname{dim} R=1$ and $R_{M}$ is an almost complete intersection for some maximal ideal $M$ of $R$, then $I^{2}$ is not primary. Explicit examples in the polynomial ring $K\left[X_{1}, X_{2}, X_{3}\right]$ over a field $K$ can easily be given. In fact, it was shown recently that for $I \in \operatorname{Spec}\left(K\left[X_{1}, X_{2}, X_{3}\right]\right)$ the ideal $I^{2}$ is primary if

Received by the editors January 9, 1978.

AMS (MOS) subject classifications (1970). Primary 13C10, 13H10; Secondary 14F10.

Key words and phrases. Almost complete intersection, conormal module, canonical module, differential module, Dedekind and Kähler different, regular differential forms. 
and only if $I$ is locally a complete intersection (J. Herzog, Ein CohenMacaulay Kriterium mit Anwendungen auf den Konormalenmodul und den Differentialmodul, Math. Z. (to appear).

Under the assumptions of the theorem condition (a) is "independent of the embedding", since condition (c) depends obviously only on $R$.

Proof of Theorem $1 .^{1}$ It is enough to prove the local version of the theorem, so we shall assume that $S$ is a regular local ring. We may also assume that $R$ is an almost complete intersection, since the theorem is known for complete intersections. Matsuoka [7] has constructed an exact sequence

$$
0 \rightarrow K_{R} \rightarrow R^{r+1} \rightarrow I / I^{2} \rightarrow 0 .
$$

Moreover, Aoyama ([1, Lemma]) has shown the formula

$$
\operatorname{depth}\left(K_{R_{P}}\right)=\operatorname{Min}\left\{2+\operatorname{depth}\left(R_{P}\right), \operatorname{dim} R_{P}\right\}
$$

for all $P \in \operatorname{Spec}(R)$.

Let $C$ be the cokernel of $\left(I / I^{2}\right)^{*} \rightarrow\left(R^{r+1}\right)^{*}$, where ${ }^{*}$ denotes the $R$-dual module. Then there is a linear map $\tau: K_{R} \rightarrow C^{*}$ such that the diagram with exact rows

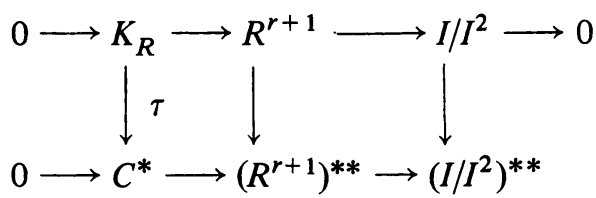

is commutative.

Suppose (a) is satisfied. Then $I / I^{2} \rightarrow\left(I / I^{2}\right)^{* *}$ is injective, since $R$ is a domain, and therefore $\tau$ is an isomorphism. Since $C^{*}$ is reflexive, being the dual of a finitely generated module over a noetherian domain, $K_{R}$ is also reflexive. If $K_{R}$ is reflexive, then so is $K_{R_{P}}$ for all $P \in \operatorname{Spec}(R)$ with ht $(P)=$ 1. By $[4,7.29] R_{p}$ has to be Gorenstein. But $R_{p}$ is an almost complete intersection or a complete intersection. By [5] only the second possibility can hold, hence (c) follows from (b).

Assume now that condition (c) of the theorem is satisfied. Then $\operatorname{dim} R_{p} \geqslant$ 2 , if $R_{p}$ is an almost complete intersection; hence $\operatorname{depth}\left(K_{R_{p}}\right) \geqslant 2$ by (2) and $\operatorname{depth}\left(R_{p} \otimes_{R} I / I^{2}\right) \geqslant 1$ by (1). Thus $P$ is not an associated prime of $I / I^{2}$. If $R_{P}$ is a complete intersection, then $R_{P} \otimes_{R} I / I^{2}$ is even free. We conclude that $I / I^{2}$ is torsion free.

2. An exact sequence for the torsion of the conormal module. The torsion $T\left(I / I^{2}\right)$ of $I / I^{2}$ is related to the Kähler and Dedekind different of $R$ over a suitable subring. In order to simplify we make the following assumptions: $S=k\left[X_{1}, \ldots, X_{n}\right]$ is a power series algebra over a perfect field $k$ and $I \in \operatorname{Spec}(S)$.

\footnotetext{
${ }^{1}$ The author wishes to thank J. Herzog, T. Matsuoka and R. Waldi for comments leading to a generalization of the original theorem and a simplified proof.
} 
In $R=S / I$ we write $x_{i}$ for the image of $X_{i}$. If $\operatorname{dim} R=d$, there is a power series algebra $Q$ of $d$ variables over $k$, such that $Q \subset R, R$ is a $Q$-module of finite type and the quotient field $L$ of $R$ is separable algebraic over the quotient field $K$ of $Q$.

After a change of variables, if necessary, we may assume that $Q=$ $k\left[x_{1}, \ldots, x_{d}\right]$. We may identify $Q$ with the subalgebra $k\left[X_{1}, \ldots, X_{d}\right]$ of $S$. Moreover we have an exact sequence

$$
0 \rightarrow T\left(I / I^{2}\right) \rightarrow I / I^{2} \rightarrow R \underset{S}{\otimes} D_{Q}(S) \rightarrow D_{Q}(R) \rightarrow 0,
$$

where $D_{Q}$ is the Kähler differential module relative to $Q$. Suppose now $I$ is an almost complete intersection of height $r=n-d$ and $\left\{F_{1}, \ldots, F_{r+1}\right\}$ a system of generators of $I$. We may assume that the mapping $\beta: R^{r+1} \rightarrow I / I^{2}$ in (1) sends the canonical basis element $e_{i}$ of $R^{r+1}$ to the image $\bar{F}_{i}$ of $F_{i}$ in $I / I^{2}(i=1, \ldots, r+1)$. Combining (1) and (3) we get a commutative diagram with exact rows and columns

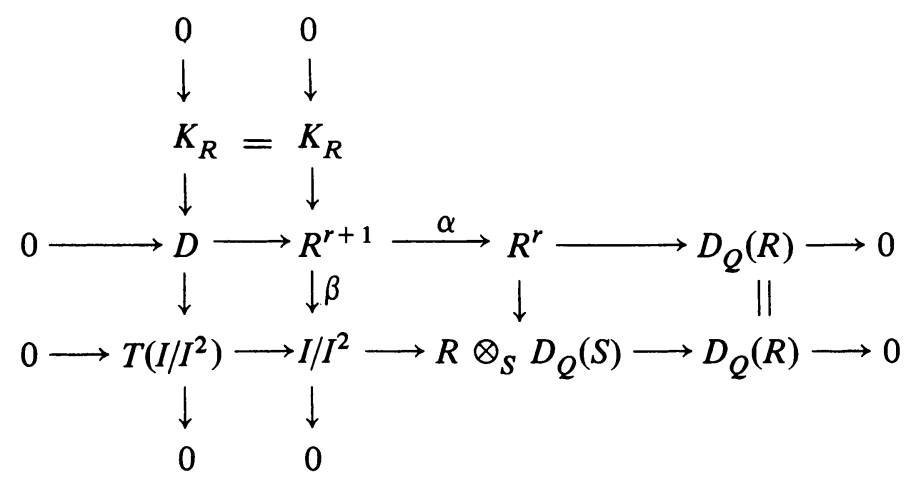

where $\alpha$ is given by the Jacobian matrix $J=\left(\partial F_{i} / \partial x_{k}\right)_{i=1, \ldots, r+1 ; k=d+1, \ldots, n}$ and $D=\operatorname{ker}(\alpha)$.

Lemma 1. $D \cong \mathscr{D}(R / Q)^{-1}$, where $\mathfrak{D}$ is the Kähler different of $R$ over $Q$, i.e. the ideal generated by all $r \times r$ minors of $J$. In particular, we have an exact sequence

$$
0 \rightarrow K_{R} \rightarrow \mathfrak{D}(R / Q)^{-1} \rightarrow T\left(I / I^{2}\right) \rightarrow 0 .
$$

Proof. By tensoring the middle row of (4) with $L$ we see that $D \otimes_{R} L \cong$ $L$. By Cramer's rule $\operatorname{ker}(\alpha \otimes L)=L \cdot\left(\Delta_{1} e_{1}+\cdots+\Delta_{r+1} e_{r+1}\right)$, where $\Delta_{1}, \ldots, \Delta_{r+1}$ are the $r \times r$ minors of $J$ (with suitable signs). $D$ can be identified with the set of all $\lambda \in L$ for which $\lambda \Delta_{i} \in R(i=1, \ldots, r+1)$, i.e. with $\mathfrak{D}(R / Q)^{-1}$.

3. Applications to differential forms. Under the assumptions as in the beginning of $\$ 2$ we consider in the $L$-vector space $\Lambda^{d}\left(L \otimes_{R} D_{k}(R)\right)$ of "meromorphic $d$-forms" the $R$-submodule

$$
\Omega_{R}^{\prime}:=\mathfrak{D}(R / Q)^{-1} d x_{1} \wedge \cdots \wedge d x_{d} .
$$


LeMma 2. $\Omega_{R}^{\prime}$ does not depend on the choice of $Q \subset R$.

Let $Q^{\prime}=k\left[y_{1}, \ldots, y_{d}\right]$ be another subalgebra of $R$ having analogous properties as $Q$. In $\Lambda^{d}\left(L \otimes_{R} D_{k}(R)\right)$ we have an equation

$$
d x_{1} \wedge \cdots \wedge d x_{d}=\delta d y_{1} \wedge \cdots \wedge d y_{d} \quad(\delta \in L \backslash\{0\})
$$

and in $\Lambda^{n}\left(L \otimes_{S} D_{k}(S)\right)$

$$
\begin{aligned}
d x_{1} \wedge \cdots & \wedge d s_{d} \wedge d F_{i_{1}} \wedge \cdots \wedge d F_{i_{r}} \\
& =\delta d y_{1} \wedge \cdots \wedge d y_{d} \wedge d F_{i_{1}} \wedge \cdots \wedge d F_{i_{r}}
\end{aligned}
$$

if $F_{i}, \ldots, F_{i_{r}}$ are taken from a set of generators $\left\{F_{1}, \ldots, F_{m}\right\}$ of $I$. From this we can conclude that $\mathfrak{D}(R / Q)=\delta \mathfrak{D}\left(R / Q^{\prime}\right)$ and $\mathfrak{D}(R / Q)^{-1} d x_{1} \wedge \cdots \wedge$ $d x_{d}=\mathfrak{D}\left(R / Q^{\prime}\right)^{-1} d y_{1} \wedge \cdots \wedge d y_{d}$.

Let $\Subset(R / Q)$ be the Dedekind complementary module of $R$ over $Q$, i.e. the set of all $\lambda \in L$ such that $\sigma_{L / K}(\lambda r) \in Q$ for all $r \in R$, where $\sigma_{L / K}: L \rightarrow K$ is the canonical trace. It is known that $\mathfrak{S}(R / Q) \subset \mathfrak{D}(R / Q)^{-1}$ and that

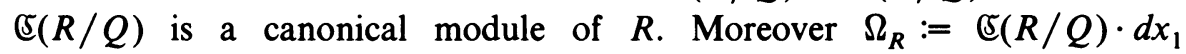
$\wedge \cdots \wedge d x_{d}$ does not depend on the choice of $Q$ (see [6]).

The $R$-modules $\Omega_{R}$ and $\Omega_{R}^{\prime}$ represent two possibilities to define "regular $d$-forms for $R$ ". A third one is given by taking the image $\Omega_{R}^{\prime \prime}$ of $\Lambda^{d} D_{k}(R)$ in $\Lambda^{d}\left(L \otimes_{R} D_{k}(R)\right)$.

If $R$ is a regular local ring, then $\Omega_{R}=\Omega_{R}^{\prime}=\Omega_{R}^{\prime \prime}$. For a complete intersection $R$ still $\Omega_{R}=\Omega_{R}^{\prime}$. The situation for almost complete intersections describes

THEOREM 2. Let $R$ be an almost complete intersection. Then

$$
\Omega_{R}^{\prime} / \Omega_{R} \cong T\left(I / I^{2}\right)
$$

Hence the following conditions are equivalent:

(a) $\Omega_{R}=\Omega_{R}^{\prime}$.

(b) $R_{P}$ is a complete intersection for all $P \in \operatorname{Spec}(R), \operatorname{ht}(P)=1$.

(c) $\Omega_{R}$ is reflexive.

Proof. We shall use the construction of the exact sequence (1) given by Matsuoka [7]. By the "Primbasissatz" there is a minimal system of generators $\left\{F_{1}, \ldots, F_{r+1}\right\}$ of $I$ such that $\left\{F_{1}, \ldots, F_{r}\right\}$ is an $S$-regular sequence and $I S_{I}=\left(F_{1}, \ldots, F_{r}\right) \cdot S_{I}$.

Let $J:=\left(F_{1}, \ldots, F_{r}\right) \cdot S$. Then $K_{R} \cong J: I / J$ as $R$-module. There is a well-defined map $\gamma: J: I \rightarrow R^{r+1}$ given as follows: For $G \in J: I$ let $-G F_{r+1}=G_{1} F_{1}+\cdots+G_{r} F_{r}\left(G_{i} \in S\right)$. Then $\gamma$ maps $G$ onto $\sum_{i=1}^{r} \bar{G}_{i} e_{i}+$ $\bar{G} e_{r+1}$, where $\bar{G}, \bar{G}_{i}$ are the images of $G, G_{i}$ in $R . \gamma$ induces an injection $J: I / J \rightarrow R^{r+1}$ whose image is the kernel of $R^{r+1} \rightarrow I / I^{2}$.

Let $\Sigma=S / J$. We can choose $Q=k\left[X_{1}, \ldots, X_{d}\right]$ such that $\Sigma$ is a $Q$-module of finite type (and $L$ separable algebraic over $K$, as before). Since 
$L$ is the residue field of $S_{l}$, we can conclude that

$$
\Delta_{r+1}=\frac{\partial\left(F_{1}, \ldots, F_{r}\right)}{\partial\left(x_{d+1}, \ldots, x_{n}\right)} \neq 0 .
$$

We use diagram (4) with the sequence $0 \rightarrow K_{R} \rightarrow R^{r+1} \rightarrow I / I^{2} \rightarrow 0$ as described above. With the notations as in the proof of Lemma 1 the mapping $D \rightarrow R^{r+1}$ identifies each $\lambda \in \mathscr{D}(R / Q)^{-1}$ with $\lambda\left(\Delta_{1} e_{1}+\cdots+\Delta_{r+1} e_{r+1}\right) \in$ $R^{r+1}$. This element is in $\operatorname{ker}(\beta)$ iff $\lambda \Delta_{r+1}$ is in the image of $J: I$ in $R$. In order to prove Theorem 2 it is therefore sufficient to show

LEMMA 3. If $\Delta$ is the image of $J: I$ in $R$, then

$$
\Delta=\frac{\partial\left(F_{1}, \ldots, F_{r}\right)}{\partial\left(x_{d+1}, \ldots, x_{n}\right)} \cdot \Im(R / Q) .
$$

Proof. Let $I^{\prime}=J: I$. We have $J=I \cap I^{\prime}$ and $I^{\prime}$ has only associated primes $P_{1}, \ldots, P_{s}$ of height $r$ and different from $I$. If $\bar{I}, \overline{I^{\prime}}$ and $\bar{P}_{i}$ $(i=1, \ldots, s)$ denote the images in $\Sigma$, then $\bar{I} \cap \overline{I^{\prime}}=(0), \overline{I^{\prime}}=\operatorname{Ann}_{\Sigma}(\bar{I})$ and $\Sigma_{\bar{I}}=L$. For the full ring of quotients of $\Sigma$ we have

$$
Q(\Sigma)=K \underset{Q}{\otimes} \Sigma=L \times \Sigma_{\bar{P}_{1}} \times \cdots \times \Sigma_{\bar{P}_{s}}
$$

The image of $\overline{I^{\prime}}$ in $Q(\Sigma)$ is $\Delta \times(0) \times \cdots \times(0)$.

In the commutative diagram of canonical homomorphisms

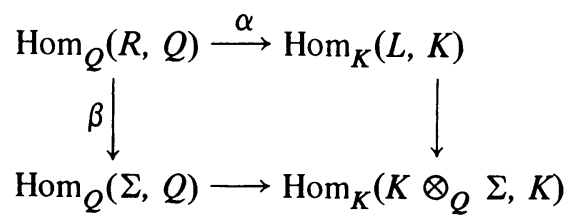

all mappings are injective. Let $\sigma_{L / K}: L \rightarrow K, \sigma_{\Sigma / Q}: \Sigma \rightarrow Q$ and $\sigma: K \otimes_{Q} \Sigma$ $\rightarrow K$ be the canonical traces. We have $\operatorname{Hom}_{K}(L, K)=L \sigma_{L / K}$ and $\operatorname{im}(\alpha)=$ $\S(R / Q) \sigma_{L / K}$. Moreover, since $\Sigma / Q$ is a complete intersection, $\operatorname{Hom}_{Q}(\Sigma, Q)$ $=\Sigma \cdot \eta$ with a trace map $\eta: \Sigma \rightarrow Q$, which by Scheja-Storch $[8,4.2]$, can be chosen in such a way that

$$
\sigma_{\Sigma / Q}=\frac{\partial\left(F_{1}, \ldots, F_{r}\right)}{\partial\left(\bar{X}_{d+1}, \ldots, \bar{X}_{n}\right)} \cdot \eta
$$

where $\partial\left(F_{1}, \ldots, F_{r}\right) / \partial\left(\bar{X}_{d+1}, \ldots, \bar{X}_{n}\right)$ denotes the image of the Jacobian determinant in $\Sigma$.

We have $\operatorname{im}(\beta)=\overline{I^{\prime}} \eta$, since for $s \in \Sigma$ the map $s \eta$ factors through $R$ iff $s \in \bar{I}^{\prime}$. From (5) we get a decomposition

$$
\begin{aligned}
\operatorname{Hom}_{K}(K \underset{Q}{\otimes} \Sigma, K)=\left(L \times \Sigma_{\bar{P}_{1}} \times \cdots \times \Sigma_{\bar{P}_{s}}\right) \cdot \eta \\
\quad=\operatorname{Hom}_{K}(L, K) \times \operatorname{Hom}_{K}\left(\Sigma_{\bar{P}_{1}}, K\right) \times \cdots \times \operatorname{Hom}_{K}\left(\Sigma_{\bar{P}_{s}}, K\right),
\end{aligned}
$$


and $\operatorname{Hom}_{K}(L, K) \rightarrow \operatorname{Hom}_{K}\left(K \otimes_{Q} \Sigma, K\right)$ is the canonical injection onto the first factor.

The image of $\overline{I^{\prime}} \eta$ in $\operatorname{Hom}_{K}\left(K \otimes_{Q} \Sigma, K\right)$ is

$$
\begin{aligned}
(\Delta \times(0) \times \cdots \times(0)) \cdot \eta & =\left[\Delta_{r+1}^{-1} \cdot(\Delta \times(0) \times \cdots \times(0))\right] \cdot \sigma \\
& =\left(\Delta_{r+1}^{-1} \cdot \Delta \cdot \sigma_{L / K}\right) \times(0) \times \cdots \times(0) .
\end{aligned}
$$

We obtain $\S(R / Q) \cdot \sigma_{L / K}=\Delta_{r+1}^{-1} \cdot \Delta \cdot \sigma_{L / K}$, which proves the claim.

If $R$ is an almost complete intersection of dimension 1 the length of $T\left(I / I^{2}\right)$ is related to the length of the torsion $T\left(D_{k}(R)\right)$ of the differential module.

Consider the exact sequence

$$
0 \rightarrow T\left(D_{k}(R)\right) \rightarrow D_{k}(R) \rightarrow \Omega_{R} \rightarrow C \rightarrow 0,
$$

where $C$ is the cokernel of the canonical map $D_{k}(R) \rightarrow \Omega_{R}$.

If $Q \subset R, Q=k[x]$ is chosen as above, then $R d x$ is a free submodule of $D_{k}(R)$ and hence we have also exact sequences

$$
0 \rightarrow T\left(D_{k}(R)\right) \rightarrow D_{k}(R) / R d x \rightarrow \Omega_{R} / R d x \rightarrow C \rightarrow 0
$$

and

$$
0 \rightarrow \Omega_{R} / R d x \rightarrow \Omega_{R}^{\prime} / R d x \rightarrow T\left(I / I^{2}\right) \rightarrow 0 .
$$

This gives us the length-formula

$$
l(C)=l\left(T\left(D_{k}(R)\right)\right)-l\left(T\left(I / I^{2}\right)\right)+l\left(\Omega_{R}^{\prime} / R d x\right)-l\left(D_{k}(R) / R d x\right) .
$$

By Berger [2, Satz 2], $l\left(D_{k}(R) / R d x\right)=l\left(\mathfrak{D}(R / Q)^{-1} / R\right)=l\left(\Omega_{R}^{\prime} / R d x\right)$, therefore

$$
l(C)=l\left(T\left(D_{k}(R)\right)\right)-l\left(T\left(I / I^{2}\right)\right) .
$$

Since $\operatorname{dim} R=1$ we have $T\left(I / I^{2}\right) \neq 0$, hence

$$
0<l\left(T\left(I / I^{2}\right)\right) \leqslant l\left(T\left(D_{k}(R)\right)\right)
$$

and

$$
l(C)<l\left(T\left(D_{k}(R)\right)\right)
$$

\section{REFERENCES}

1. Y. Aoyama, A remark on almost complete intersections, Manuscripta Math. 22 (1977), 225-228.

2. R. Berger, Differentialmoduln eindimensionaler lokaler Ringe, Math. Z. 81 (1963), 326-354.

3. D. Ferrand, Suite regulière et intersection complète, C. R. Acad. Sci. Paris Sér. A-B 264 (1967), 427-428.

4. J. Herzog and E. Kunz, Der kanonische Modul eines Cohen-Macaulay-Rings, Lecture Notes in Math., vol. 238, Springer-Verlag, Berlin, 1971.

5. E. Kunz, Almost complete intersections are not Gorenstein rings, J. Algebra 28 (1974), $111-115$. 
6. __ Differentialformen auf algebraischen Varietäten mit Singularitäten. II, Abh. Math. Sem. Univ. Hamburg 47 (1978), 42-70.

7. T. Matsuoka, On almost complete intersections, Manuscripta Math. 21 (1977), 329-340.

8. G. Scheja and U. Storch, Uber Spurfunktionen bei vollständigen Durchschnitten, J. Reine Angew. Math. 278/279 (1975), 174-190.

9. W. Vasconcelos, Ideals generated by R-sequences, J. Algebra 6 (1967), 309-316.

Fachibreich Mathematix, Universität Regensburg, D 8400 Regensburg, Federal REPUBlic OF Germany 